\title{
Qualidade de vida dos cuidadores de crianças com transtorno do espectro autístico
}

\author{
Quality of life of autistic children's caretakers
}

\author{
Milene Rossi Pereira Barbosa ${ }^{1}$, Fernanda Dreux Miranda Fernandes ${ }^{2}$
}

\begin{abstract}
RESUMO
Objetivo: Avaliar a qualidade de vida dos cuidadores de crianças incluídas no espectro autístico e determinar se há algum tipo de relação entre os diferentes domínios e aspectos demográficos como escolaridade e classe social. Métodos: Foram sujeitos 150 cuidadores de crianças pertencentes ao espectro autístico, com idades entre 24 e 65 anos, os quais responderam as questões feitas por meio do instrumento de avaliação da qualidade de vida WHOQOL-bref, elaborado pelo Programa de Saúde Mental da Organização Mundial de Saúde, composto de 26 questões, divididas em quatro domínios (Físico, Psicológico, Relações Sociais, e Meio Ambiente). Resultados: Os dados revelaram diferenças significantes entre todos os domínios. O único domínio do questionário de qualidade de vida que mostrou correlação estatisticamente significante com todas as variáveis demográficas foi o domínio Meio Ambiente. Conclusão: Fatores como acesso a lazer, saúde e transporte têm papel importante na qualidade de vida percebida por pais de crianças do espectro autístico.
\end{abstract}

Descritores: Qualidade de vida; Transtorno autístico; Família; Cuidadores; Assistência ao paciente; Criança; Adolescente

\section{INTRODUÇÃO}

A presença da criança autista tende a modificar as relações entre os familiares. Os sintomas do distúrbio desencadeiam elevados níveis de estresse nos membros da família ${ }^{(1)}$. As relações sociais das famílias com crianças autistas ficam embaraçosas e se reduzem, podendo até haver rupturas em seus vínculos sociais. Frequentemente elas passam a ocupar uma posição inferior àquela que desfrutavam anteriormente na sociedade.

Poucos são os estudos a respeito da qualidade de vida dos familiares das crianças com autismo ${ }^{(2)}$. Pais de autistas relatam passar por períodos de estresse pela dificuldade de comunicação das crianças, do comportamento, do isolamento social nas atividades de vida diária e pela falta de entendimento pela comunidade ${ }^{(1)}$.

As crises psicológicas em geral se dão por depressão e ansiedade, sendo estas maiores do que em outras doenças ${ }^{(3)}$. Os três mais importantes fatores causadores do estresse dos

Trabalho realizado no Departamento de Fisioterapia, Fonoaudiologia e Terapia Ocupacional da Faculdade de Medicina da Universidade de São Paulo - USP - São Paulo (SP), Brasil.

(1) Pós-graduanda (Mestrado) em Ciências da Reabilitação pela Faculdade de Medicina da Universidade de São Paulo - USP - São Paulo (SP), Brasil.

(2) Livre-docente, Professora Associada do Departamento de Fisioterapia, Fonoaudiologia e Terapia Ocupacional da Faculdade de Medicina da Universidade de São Paulo - USP - São Paulo (SP), Brasil.

Endereço para correspondência: Milene Rossi Pereira Barbosa. R. Miguel

Consentino, 128, Jd. Santa Tereza, Jundiaí (SP), Brasil, CEP: 13211-407.

E-mail: milene@usp.br

Recebido em: 2/4/2009; Aceito em: 5/8/2009 pais são: a pouca aceitação dos comportamentos autísticos pela sociedade e por outros membros da família, a manutenção necessária e a ausência de suporte social ${ }^{(4)}$. As características negativas associadas com as inabilidades da criança frequentemente também aumentam os níveis de estresse ${ }^{(5)}$.

O que se observa, na maioria dos estudos americanos publicados até hoje a respeito do estresse dos pais de autistas, é que esses são limitados pelo pequeno tamanho da amostra, pela baixa significância estatística e pelo fato de as amostras serem extraídas de relatos de escolas, clínicas e organizações de pais ${ }^{(3)}$.

Há um grande número de fatores de impacto que intensificam os níveis de estresse, como a severidade dos casos, a dificuldade de acesso aos serviços especiais de que necessitam e fatores econômicos e culturais ${ }^{(6)}$. $\mathrm{O}$ recebimento ou não de suporte informal acaba por influenciar a qualidade de vida tanto do autista como de seus familiares ${ }^{(7)}$.

Frequentemente a qualidade de vida dos cuidadores de autistas é moderada pelas condições socioeconômicas, pelo suporte social e características dos pais e das crianças ${ }^{(8-9)}$. Por exemplo, o problema da criança em não dormir frequentemente causa exaustão nos pais, o que acaba refletindo em seu trabalho ${ }^{(10)}$.

A busca de um instrumento que avaliasse qualidade de vida dentro de uma perspectiva internacional fez com que a Organização Mundial da Saúde organizasse um projeto colaborativo multicêntrico. $\mathrm{O}$ resultado deste projeto foi a elaboração do WHOQOL-100, um instrumento de avaliação de qualidade de vida composto por 100 itens $^{(11)}$. 
A necessidade de instrumentos curtos que demandem pouco tempo para seu preenchimento, mas com características psicométricas satisfatórias, fez com que o Grupo de Qualidade de Vida da OMS desenvolvesse uma versão abreviada do WHOQOL-100, o WHOQOL-bref ${ }^{(12)}$ (WHOQOL Group, 1998) composto de 26 questões, divididas em quatro domínios (Físico, Psicológico, Relações Sociais e Meio Ambiente).

As questões do WHOQOL-bref foram formuladas para uma escala de respostas do tipo Likert, com uma escala de intensidade (variando de nada a extremamente), capacidade (variando de nada a completamente), frequência (variando de nunca a sempre) e avaliação (variando de muito insatisfeito a muito satisfeito ou muito ruim a muito bom).

Uma pesquisa americana ${ }^{(13)}$, realizada com o objetivo de investigar a qualidade de vida de cuidadores de autistas utilizou como método a aplicação do instrumento WHOQOL-bref. Nos resultados, os pais mostraram escores mais baixos nos domínios de relação social do que pais de crianças normais. As mães mostraram escores mais baixos nos domínios: físico e de relação social do que mães de crianças normais e, ainda, mais baixos no domínio psicológico do que mães de deficientes mentais. Neste estudo, foi concluído que pais de crianças com autismo necessitam de suportes e intervenções mais específicos.

Quando a criança tem atendimentos com especialistas e suporte apropriado, as oportunidades de viver independentemente são maiores ${ }^{(14)}$, assim como as situações de trabalho e relações sociais ${ }^{(15)}$, minimizando os níveis de estresse dos pais $^{(16)}$. A minoria dos autistas de alto-funcionamento adultos que moram nos Estados Unidos relata que moram sozinhos, tiveram oportunidades de estudo, foram capazes de fazer amigos sozinhos, casaram-se ou têm emprego. Quando o QI situa-se acima de 70 eles são mais queridos pela sociedade, porém as inabilidades cognitivas (déficit fundamental no autismo) deixam-nos isolados ${ }^{(17)}$.

O objetivo do presente estudo foi avaliar a qualidade de vida dos cuidadores de crianças incluídas no espectro autístico e determinar se há algum tipo de relação entre os diferentes domínios e aspectos demográficos de escolaridade dos pais e classe social.

\section{MÉTODOS}

Essa pesquisa foi encaminhada à comissão de ética da Faculdade de Medicina da Universidade de São Paulo (FMUSP) e recebeu aprovação, sob o protocolo no 1155/06.

Participaram deste estudo, os cuidadores principais de 150 crianças e adolescentes com diagnósticos incluídos no Espectro Autístico, atribuídos por médicos neurologistas e/ ou psiquiatras segundo critérios do $\mathrm{DSM}^{(18)}$ ou da CID-10 ${ }^{(19)}$.

Os referidos pais têm entre 24 e 65 anos de idade e seus filhos estão inseridos em dois tipos de atendimento: atendimento institucional (83 sujeitos) e atendimento ambulatorial (67 sujeitos). Todos residem em regiões metropolitanas com mais de um milhão de habitantes na região sudeste do Brasil.

Os questionários WHOQOL-bref foram respondidos em entrevistas individuais da primeira autora com os sujeitos, realizadas nos locais de atendimento das crianças e tiveram duração média de 50 minutos.

A fim de verificar se os diferentes domínios que compõem o questionário referente à qualidade de vida dos respectivos cuidadores se diferenciam entre si foi aplicada a Análise de Variância (ANOVA).

Considerando que todos os domínios diferem entre si, foi aplicado o Test t de Student a fim de verificar quais eram os pares de variáveis responsáveis pela significância estatística encontrada na ANOVA.

Com o intuito de verificar o grau de relacionamento entre as variáveis: escolaridade de pai e mãe e classe econômica, pertencentes a sua relação com os domínios avaliados durante a aplicação do questionário de qualidade de vida, foi aplicada a Análise de Correlação de Spearman.

\section{RESULTADOS}

Os 150 questionários incluíam questões sócio-demográficas sobre ambos os pais. Dessa forma, 39\% das mães são analfabetas, $18 \%$ tem o ensino fundamental completo, $34 \%$ tem o ensino médio completo e apenas $8 \%$ tem o ensino superior completo. Trinta e oito por cento dos pais são analfabetos, $20 \%$ tem o ensino fundamental completo, $45 \%$ o ensino médio e $6 \%$ o ensino superior completo. Esses dados estão sintetizados no Quadro 1.

Quadro 1. Nível de escolaridade dos pais

\begin{tabular}{|lcc|}
\hline Nível de escolaridade & Mãe & Pai \\
\hline Analfabetos & 59 & 57 \\
Ensino fundamental completo & 27 & 32 \\
Ensino médio completo & 51 & 45 \\
Ensino superior completo & 13 & 09 \\
\hline
\end{tabular}

Quanto à classe econômica, 15,3\% pertencem à classe D (573 reais mensais), 24\% à classe C2 (861 reais mensais), $31,3 \%$ à classe $\mathrm{C} 1$ (1.318 reais mensais), $15,3 \%$ à classe $\mathrm{B} 2$ (2.256 reais mensais), $10 \%$ à classe $\mathrm{B} 1$ (3.994 reais mensais), $3,33 \%$ à classe $\mathrm{A} 2$ (7.557 reais anuais) e apenas $0,66 \%$ à classe A1(14.250 reais anuais). O Quadro 2 apresenta esses dados.

Quadro 2. Classe econômica das famílias estudadas segundo critérios da Associação Brasileira de Empresas de Pesquisa

\begin{tabular}{|ll|}
\hline Classe econômica & $\mathrm{N}$ \\
\hline Classe A1 & 01 \\
Classe A2 & 05 \\
Classe B1 & 15 \\
Classe B2 & 23 \\
Classe C1 & 47 \\
Classe C2 & 36 \\
Classe D & 23 \\
Classe E & 00 \\
\hline
\end{tabular}

* Fonte: http://www.abep.org/default.aspx?usaritem=arquivos\&iditem=23

A comparação entre os diversos domínios identificou diferenças estatisticamente significantes entre eles (Tabela 1). 
Tabela 1. Diferenças entre os domínios do WHOQOL-bref (ANOVA)

\begin{tabular}{lcccc}
\hline Domínios & $\mathrm{N}$ & Média & Variância & Valor de $\mathrm{p}$ \\
\hline Psicológico & 150 & 31,3 & 15,5 & \\
Físico & 150 & 8,9 & 2,5 & $>0.001$ \\
Meio ambiente & 150 & 34,5 & 28,9 & \\
Relações sociais & 150 & 10,3 & 4,8 & \\
\hline
\end{tabular}

A comparação entre todos os domínios revelou diferenças significantes entre todos eles, como fica evidenciado na Tabela 2.

A Tabela 3 verifica a correlação entre cada um dos domínios e as variáveis sócio-demográficas. Os dados apontam para diferenças significantes apenas em relação ao domínio Meio Ambiente, que inclui informações referentes ao lazer, ao acesso aos serviços de saúde e transporte e às condições de moradia. Essas relações foram significantes para todas as variáveis demográficas (classe sócio-econômica e escolaridade paterna e materna).

\section{DISCUSSÃO}

Os dados apresentados podem ser significativos na determinação de procedimentos para a melhoria da qualidade de vida dos cuidadores de crianças inseridas no espectro autístico. Esta inclui uma variedade potencial maior de condições que podem afetar a percepção do indivíduo, seus sentimentos e comportamentos relacionados com o seu funcionamento diário, incluindo, mas não se limitando, à sua condição de saúde e às intervenções médicas ${ }^{(20)}$.

A literatura aponta que as questões de comunicação, comportamento e atividades diárias são fatores desencadeantes do estresse familiar ${ }^{(1)}$; nesta pesquisa, por meio do WHOQOLbref, estas e outras questões foram avaliadas. A análise estatística revelou que não somente estes aspectos (incluídos nos domínios físico, psicológico e de relações sociais, segundo o questionário), mas questões sócio-demográficas também foram determinantes na caracterização da qualidade de vida dos sujeitos da pesquisa.

Os resultados dessa pesquisa mostram que o instrumento utilizado, apesar do número reduzido de questões, apresenta boas características psicométricas, pois identifica diferenças estatisticamente significantes entre os domínios.

A diferença estatisticamente significante encontrada no domínio Meio Ambiente, quando comparada com as demais variáveis referentes às questões socio-demográficas parece indicar o resultado desse processo, como relatado na literatura $^{(6)}$. Fatores referentes ao domínio Meio Ambiente, como as dificuldades no acesso aos serviços de saúde, contribuem para o aumento do nível de estresse dos cuidadores de crianças e adolescentes incluídos no espetro autístico. Além disso, frequentemente a qualidade de vida é mediada por questões socioeconômicas ${ }^{(8)}$.

Uma vez que o domínio Meio Ambiente inclui questões referentes ao lazer e à educação, e corroborando os achados desse estudo, pesquisas internacionais ${ }^{(21)}$ mostram relatos de pais e profissionais a respeito da falta de informação a respeito de como encontrar lazer e educação disponível para os portadores do autismo, o que acaba intensificando os níveis de estresse dos cuidadores e interferindo diretamente na sua qualidade de vida.

Algumas limitações do presente estudo devem se consideradas, como por exemplo, o fato de a maioria dos estudos que abordam o assunto serem de origem norte-americana, o que

Tabela 2. Comparação entre os domínios do WHOQOL-bref (Teste t Student)

\begin{tabular}{lcccccccccccc}
\hline Medidas estatísticas & Psi & Fis & Psi & MA & Psi & RS & Fis & MA & Fis & RS & MA & RS \\
\hline $\mathrm{N}$ & 150 & 150 & 150 & 150 & 150 & 150 & 150 & 150 & 150 & 150 & 150 & 150 \\
Média & 31,3 & 8,9 & 31,3 & 34,5 & 31,3 & 10,3 & 8,9 & 34,5 & 8,9 & 10,3 & 34,5 & 10,3 \\
Variância & 15,5 & 2,5 & 15,5 & 26,9 & 15,5 & 4,8 & 2,5 & 28,9 & 2,5 & 4,8 & 28,9 & 4,8 \\
\hline Valor de $p$ & $>0,001$ & $>0,001$ & $>0,001$ & & $>0,001$ & & $>0,001$ & $>0,001$ \\
\hline
\end{tabular}

Legenda: Psi = psicológico; Fis = físico; RS = relações sociais; $\mathrm{MA}$ = meio ambiente

Tabela 3. Comparação entre fatores demográficos e os domínios de qualidade de vida

\begin{tabular}{|c|c|c|c|c|c|}
\hline Variável & Estatística & Psi & Fis & MA & RS \\
\hline \multirow[t]{3}{*}{ Classe } & Coeficiente de Correlação & 0,087 & 0,043 & 0,531 & 0,087 \\
\hline & Significância (p) & 0,292 & 0,602 & $<0,001^{*}$ & 0,291 \\
\hline & $\mathrm{N}$ & 150 & 150 & 150 & 150 \\
\hline \multirow[t]{3}{*}{ Escolaridade mãe } & Coeficiente de Correlação & $-0,072$ & $-0,015$ & 0,286 & 0,023 \\
\hline & Significância (p) & 0,383 & 0,857 & $<0,001^{*}$ & 0,776 \\
\hline & $\mathrm{N}$ & 150 & 150 & 150 & 150 \\
\hline \multirow[t]{3}{*}{ Escolaridade pai } & Coeficiente de Correlação & 0,065 & 0,015 & 0,423 & 0,052 \\
\hline & Significância (p) & 0,437 & 0,860 & $<0,001^{*}$ & 0,539 \\
\hline & $\mathrm{N}$ & 143 & 143 & 143 & 143 \\
\hline
\end{tabular}

Legenda: Psi = psicológico; Fis = físico; RS = relações sociais; $M A$ = meio ambiente

${ }^{*}$ Valores estatisticamente significantes 
provavelmente amplia diferenças sócio-culturais observadas nos resultados do questionário.

Outros estudos usando o mesmo instrumento em entrevistas com pais de crianças com outros distúrbios, ou com desenvolvimento normal, poderiam fornecer informações para a identificação de fatores mais predominantes em situações específicas em ou populações distintas, o que poderia contribuir para a proposição de programas institucionais, ações de apoio e prevenção e redes de suporte terapêutico.

\section{CONCLUSÃO}

O objetivo deste estudo foi avaliar a qualidade de vida dos cuidadores de crianças incluídas no espectro autístico e determinar se há algum tipo de relação entre os diferentes domínios e aspectos demográficos como escolaridade dos pais e classe social.

Os quatro domínios (Físico, Psicológico, Relações Sociais, e Meio Ambiente) referentes ao WHOQOL-bref diferem entre si e a única variável que mostrou correlação estatisticamente significante com todas as variáveis referentes a aspectos sócio-demográficos foi o domínio Meio Ambiente o qual traz questões referentes ao lazer, ao acesso ao serviço de saúde, ao transporte e às condições de moradia.

\section{AGRADECIMENTOS}

À Fundação de Amparo à Pesquisa do Estado de São Paulo (FAPESP), pelo auxílio concedido para realização da pesquisa.

\begin{abstract}
Purpose: To assess the quality of life of autistic spectrum children's caretakers and to determine whether there is some kind of relation among the different domains and demographic aspects such as educational and social level. Methods: Subjects were 150 caretakers of children within the autistic spectrum, with ages ranging from 24 to 65 years, who answered to the WHOQOL-bref protocol, proposed by the Mental Health Program of the World Health Organization, which has 26 questions divided into four domains (Physical, Psychological, Social Relations, and Environment). Results: Data revealed significant differences among all the domains. The only domain of the quality of life questionnaire that had statistically significant correlations with all demographic variables was Environment. Conclusion: Factors such as access to leisure, health and transportation play an important role in the quality of life realized by parents of autistic spectrum children.
\end{abstract}

Keywords: Quality of life; Autistic disorder; Family; Caregivers; Patient care; Child; Adolescent

\title{
REFERÊNCIAS
}

1. Sivberg B. Family system and coping behaviours: a comparison between parents of children with autistic spectrum disorders and parents with non-autistic children. Autism. 2002;6(4):397-409.

2. Donovan AM. Family stress and ways of coping with adolescents who have handicaps: maternal perceptions. Am J Ment Retard. 1988;92(6):502-9.

3. Schieve LA, Blumberg SJ, Rice C, Visser SN, Boyle C. The relationship between autism and parenting stress. Pediatrics. 2007;119 Suppl 1:114-21.

4. Sharpley C, Bitsika V, Efremidis B. Influence of gender, parental health, and perceived expertise of assistance upon stress, anxiety, and depression among parents of children with autism. J Intellect Dev Disabil. 1997;22(1):19-28.

5. Sanders JL, Morgan SB. Family stress and adjustment as perceived by parents of children with autism or Down syndrome: implications for intervention. Child Fam Behav Ther. 1997;19(4):15-32.

6. Yeargin-Allsopp M, Rice C, Karapurkar T, Doernberg, N, Boyle, C, Murphy C. Prevalence of autism in a US metropolitan area. JAMA. 2003; 289(1):49-55.

7. Hansson L, Sandlund M, Bengtsson-Tops A, Bjarnason O, Karlsson, $\mathrm{H}$, Mackeprang $\mathrm{T}$, et al. The relationship of needs and quality of life in persons with schizophrenia living in the community. A Nordic multicenter study. Nord J Psychiatry. 2003;57(1):5-11.

8. Raina P, O’Donnell M, Schwellnus H, Rosenbaum P, King G, Brehaut $\mathrm{J}$, et al. Caregiving process and caregiver burden: conceptual models to guide research and practice. BMC Pediatr [internet]. 2004 Jan [cited 2009 Jan 9]; 4:1. Available from: http://www.ncbi.nlm.nih.gov/pmc/ articles/PMC331415/?tool=pubmed
9. Vitaliano PP, Zhang J, Scanlan JM. Is caregiving hazardous to one's physical health? A meta-analysis. Psychol Bull. 2003;129(6):946-72.

10. Benderix Y; Nordstrom B, Sivberg B. Parents' experience of having a child with autism and learning disabilities living in a group home: a case study. Autism. 2006;10(6):629-41.

11. Organização Mundial de Saúde. Divisão de Saúde Mental. Grupo WHOQOL. Versão em português dos instrumentos de avaliação de qualidade de vida (WHOQOL) 1998. Instruções de aplicação dos instrumentos WHOOQL (100 E BREVE) [internet] 1998 Set [citado 2009 Out 22]. Disponível em: http://www.ufrgs.br/psiq/whoqol-100.html

12. Organização Mundial de Saúde: Divisão de Saúde Mental, Grupo WHOQOL, versão em português dos instrumentos de avaliação de qualidade de vida (WHOQOL) 1998. Procedimentos de aplicação do WHOQOL-100 e do WHOQOL-Bref [internet] 1998 Set [citado 2009 Out 22]. Disponível em:http://www.ufrgs.br/Psiq/whoqol4.html

13. Mugno D, Ruta L, D'Arrigo VG, Mazzone L. Impairment of quality of life in parents of children and adolescents with pervasive developmental disorder. Health Qual Life Outcomes. 2007; 5: 22-9.

14. Tantam D. Psychological disorder in adolescents and adults with Asperger syndrome. Autism. 2000; 4 (1):47-62.

15. Hurlbutt K, Chalmers L. Employment and adults with Asperger syndrome. Focus Autism Other Dev Disabl. 2004; 19(4):215-22.

16. Tantam D. The challenge of adolescents and adults with Asperger syndrome. Child Adolesc Psychiatr Clin N Am. 2003; 12(1):143-63.

17. Renty J, Roeyers H. Quality of life in high-functioning adults with autism spectrum disorder: The predictive value of disability and support characteristics. Autism. 2006; 10(5):511-24. 
18. American Psychiatry Association. DSM-IV-TR. Diagnostic and statistical manual of mental disorders. 4th ed. Washington (DC): American Psychiatry Publishing; 2001.

19. Organização Mundial de Saúde. CID-10 Critérios diagnósticos para pesquisa (Classificação de transtornos mentais e de comportamento da CID-10). Porto Alegre: Artes Médicas; 1998.
20. Bullinger M, Anderson R, Cella D, Aaronson N. Developing and evaluating cross-cultural instruments from minimum requirements to optimal models. Qual Life Res. 1993; 2(6):451-9.

21. Kraus MW, Gulley S, Sciegaj M, Wells N. Access to specialty medical care for children with mental retardation, autism, and other special health care needs. Mental Retard. 2004; 41(5):329-39. 